\title{
Prophylactic negative-pressure wound therapy after ileostomy reversal for the prevention of wound healing complications in colorectal cancer patients: a randomized controlled trial
}

\author{
M. Wierdak ${ }^{1} \cdot$ M. Pisarska-Adamczyk ${ }^{1} \cdot$ M. Wysocki ${ }^{1} \oplus \cdot$ P. Major $^{1} \cdot$ K. Kołodziejska ${ }^{1} \cdot$ M. Nowakowski $^{1}$. \\ T. Vongsurbchart ${ }^{1} \cdot$ M. Pędziwiatr ${ }^{1}$
}

Received: 5 July 2020 / Accepted: 26 October 2020 / Published online: 7 November 2020

(c) The Author(s) 2020

\begin{abstract}
Background The aim of this study was to assess the usefulness of protective negative-pressure wound therapy (NPWT) in the reduction of wound healing complications (WHC) and surgical site infections (SSI) after diverting ileostomy closure in patients who underwent surgery for colorectal cancer.

Methods In this prospective randomized clinical trial in a tertiary academic surgical center, patients who had colorectal cancer surgery with protective loop ileostomy and were scheduled to undergo ileostomy closure with primary wound closure from January 2016 to December 2018 were randomized to be treated with or without NPWT. The primary endpoint was the incidence of WHC. Secondary endpoints were incidence of SSI, length of postoperative hospital stay (LOS), and length of complete wound healing $(\mathrm{CWH})$ time.

Results We enrolled 35 patients NPWT (24 males [68.6\%]; mean age 61.6 \pm 11.3 years), with NPWT and 36 patients (20 males [55.6\%]; mean age $62.4 \pm 11.3$ years) with only primary wound closure (control group). WHC was observed in 11 patients $(30.6 \%)$ in the control group and $3(8.57 \%)$ in the NPWT group $(p=0.020)$. Patients in the NPWT group had a significantly lower incidence of SSI (2 [5.71\%] vs. 8 [22.2\%] in the control group; $p=0.046)$ as well as significantly shorter median CWH (7 [7-7] days vs. 7 [7-15.5] days, $p=0.030$ ). There was no difference in median LOS between groups (3 [2.5-5] days in the control group vs. 4 [2-4] days in the NPWT group; $p=0.072$ ).

Conclusions Prophylactic postoperative NPWT after diverting ileostomy closure in colorectal cancer patients reduces the incidence of WRC and SSI.

Clinical trial registration clinicaltrials.gov (NCT04088162).
\end{abstract}

Keywords Prophylactic negative pressure wound therapy · Ileostomy closure · Prevention of wound-related complications · Colorectal cancer operation $\cdot$ Randomized controlled trial

M. Wysocki

m.wysocki@doctoral.uj.edu.pl

M. Wierdak

mateusz.wierdak@uj.edu.pl

M. Pisarska-Adamczyk

magdalena.pisarska@doctoral.uj.edu.pl

P. Major

piotr.major@uj.edu.pl

K. Kołodziejska

kat.truszkiewicz@gmail.com

\author{
M. Nowakowski \\ m.nowakowski@uj.edu.pl \\ T. Vongsurbchart \\ tanawatv19@gmail.com
}

M. Pędziwiatr

michal.pedziwiatr@uj.edu.pl

1 2nd Department of General Surgery, Jagiellonian University

Medical College, Jakubowskiego 2 Str., 30-688 Krakow,

Poland 


\section{Introduction}

Surgeries for colorectal cancer, especially procedures involving the lower rectum, are associated with a very high percentage of complications [1-3]. Despite the current recomsmendation for primary anastomosis after a resection procedure, such treatment is associated with a significant risk of anastomotic leak (up to $20 \%=[4,5]$ ). One way to reduce the risk of leak is to use a diverting ileostomy $[6,7]$. Although this is currently considered a standard treatment, especially in the group of patients undergoing neoadjuvant radiotherapy or chemoradiotherapy, the technique still has its drawbacks, such as the necessity to perform an additional surgical operation for ileostomy closure, which has a high risk of wound healing complications, particularly surgical site infections (SSI) $[8,9]$. To reduce the frequency of these complications, novel guidelines published in 2017 recommend using the purse-string suture technique [9-11]. However, this has been associated with as much as a fivefold increase in healing time, as well as less desirable cosmetic effects, in patients without SSI [12, 13]. Negative-pressure wound therapy (NPWT) is currently a widely used method of treatment in various types of infectious complications, potentially providing the opportunity to prevent infectious complications after surgery $[14,15]$ by combining the benefits of both postoperative wound closure techniques: reduced healing time, compared to primary closure, and reduced risk of infectious complications, compared to purse-string techniques.

The aim of this randomized controlled trial was to assess the usefulness of postoperative NPWT in the reduction of postoperative wound-healing complications (WHC) and SSI after diverting ileostomy closure in patients who underwent colorectal resection for cancer.

\section{Materials and methods}

\section{Patients}

A randomized controlled trial was conducted between January 2016 and December 2018 in a tertiary referral center, University Hospital (Krakow, Poland). The trial was registered at clinicaltrials.gov (NCT04088162) after approval of the protocol by ethics committee of Jagiellonian University Medical College (\#1072.6219.263.2019). The trial was designed as a single-center, randomized controlled, superiority trial with two parallel intervention arms.

\section{Participants}

Patients aged $\geq 18$ years with a history of surgery for colorectal cancer, including formation of the protective ileostomy, who were scheduled to undergo ileostomy closure as an elective procedure, were randomly divided into two groups: Group 1 to undergo postoperative NPWT and Group 2, a control group to undergo customary care (without postoperative NPWT). Patients were enrolled after providing informed consent on admission. Exclusion criteria were emergency/urgent operation, active infection, operations other than ileostomy closure, or parastomal hernioplasty. Patients who required a second operation or transfer to the intensive care unit or other hospital wards because of noninfectious complications within the first week after surgery were also excluded from the analysis.

\section{Randomization}

The 1:1 randomization with concealment was achieved using a random number generator (even/odd) [16]. Until the end of the operation, patients did not know to which group they were assigned. The randomization process and assignment of the patients to the groups were performed by a trial researcher who was not directly involved in the operation or postoperative care of the patient. Operating surgeons were also blinded to the randomization. The NPWT dressing was set up at the end of the operation in sterile conditions in the operating room by one and the same person (the designated surgeon, a member of the research team) not directly involved in the operation or postoperative patient care.

\section{Sample size calculation}

Our previous observations found the primary endpoint incidence in our population to be $33 \%$, which was consistent with the data available in the literature [8,9]. Additionally, a previous pilot study demonstrated that NPWT had decreased the incidence of WHC by 70-85\% [17]. To demonstrate that NPWT decreased WHC by $80 \%$, a total sample size of 70 subjects was needed for an alpha of 0.05 and $80 \%$ power. Thus, with expectations of omissions, we sought a total sample size of 38 patients in each arm.

\section{Procedures}

Patients' demographics, possible SSI risk factors, including age, sex, body mass index (BMI), active smoking, preoperative immunosuppressive treatment, incidence of comorbidities, amount of intraoperative bleeding, and surgery duration were prospectively collected.

\section{Surgical technique}

All patients enrolled in the study had previously undergone a resection procedure for colorectal cancer with the simultaneous formation of a diverting loop ileostomy $20-30 \mathrm{~cm}$ proximally from the ileocecal valve at the ileal loop, which 
was delivered through a circular incision on the right lower abdominal wall without mesenteric torsion. Ileostomy closure, as a second operation, was performed approximately 6 months after initial surgery, after adjuvant chemotherapy (if necessary). Patients with American Joint Committee on Cancer-AJCC—stage 0 or 1 had the ileostomy removal procedure performed much earlier, as early as 14 days, after the histopathological examination results were obtained. At ileostomy closure, a circumferential incision around the ileostomy was performed. Adhesions were gently detached from the abdominal wall with scissors. After small bowel mobilization, the short-segment small bowel resection (approximately $15-25 \mathrm{~cm}$ ) was performed. Anastomosis was performed via end-to-end single polydioxanone (PDS) Plus 4-0 running suture. Wound closure was done along three layers, including the peritoneum layer, rectus abdominis fascia, and subcutaneous layer. $2-0$ absorbable PDS Plus running suture was used for peritoneum and fascia layer, and 3-0 Vicryl Plus single sutures were used to close the subcutaneous layer. In the five cases of parastomal hernia (three in the NPWT group and two in the control group) it was necessary to perform hernia repair using a polypropylene mesh, as in the sub-lay method. In those patients, the silicone drain was placed in the subfascial layer and removed at 2 or 3 postoperative days (after exudation reduction to less than $30 \mathrm{ml} /$ day). In the control group, the skin was closed by six to eight single non-absorbable Monosyn 3-0 loose sutures every 7-9 $\mathrm{mm}$, and a sterile wound dressing was placed. In the NPWT group, the skin was closed with 4-6 single non-absorbable Monosyn 3-0 loose sutures every $1 \mathrm{~cm}$. A NANOVA negative-pressure dressing was placed over the entire length of the incision. Thanks to the use of NPWT, which stabilizes wound edges, we were able to place less skin sutures without the risk of wound dehiscence. NPWT also provides the opportunity to evacuate exudate from the subcutaneous tissue in sterile conditions and prevent the formation of a seroma or hematoma. In the control group, the first dressing change was made $48 \mathrm{~h}$ after the operation, and thereafter dressings were changed daily until the removal of sutures on postoperative day 7. In the NPWT group, the NANOVA dressing was taken out at $72 \mathrm{~h}$. Three Steri-Strips were placed between the sutures, and a standard sterile dressing was placed. The dressing was then changed every $24 \mathrm{~h}$ until the removal of sutures on postoperative day 7 .

\section{Perioperative care}

All patients received second-generation cephalosporin $(20 \mathrm{mg} / \mathrm{kg}$ ) $30 \mathrm{~min}$ before the incision. On postoperative day 1 , patients were fully mobilized and received a standard oral liquid diet with a volume restriction of 11 . Patients with good diet tolerance were fed with a standard hospital diet from postoperative day 2. Perioperative care of patients in this study was compliant with the enhanced recovery after surgery (ERAS) protocol in colorectal surgery [18].

Healing was evaluated during dressing changes in the ward and then during routine check-ups in the outpatient clinic on postoperative days 7 and 14. Patients who noticed any abnormalities related to wound healing contacted the outpatient clinic by telephone and were admitted for an additional visit. After 30 days, the patients were contacted by telephone to obtain information about possible abnormalities related to healing and were asked to send a photo of the healed wound by e-mail. ForWHC, the frequency of monitoring visits was based on clinical status. The last visit to the outpatient clinic was made approximately 2 weeks after $\mathrm{CWH}$.

\section{End point criteria}

The primary endpoint was the reduction of WHC after protective ileostomy closure. WHC were defined as any condition of the wound that required postoperative intervention other than a change of dressing or removal of stiches.

Secondary endpoints were the incidence of SSI, postoperative length of hospital stay (LOS) and the duration of CWH. CWH was defined as complete closure of the wound without any secretion from the wound, as assessed at the outpatient clinic or reported by the patient. Incisional SSI diagnosis were made according to the criteria of the Center for Disease Control (CDC) and European Centre for Disease Prevention and Control (ECDC) for diagnosis of SSI [19, 20].

\section{Statistical analysis}

Continuous data are presented as the medians and interquartile ranges, unless otherwise indicated. Continuous variables were compared using the Mann-Whitney test and Student's $t$-test. Categorical variables were compared using the chi-square test, including Yates' correction or Fisher's exact test when necessary. The level of significance was set at $p<0.05$. Logistic regression models were used to detect possible risk factors for WHC and SSI incidence. In the case of LOS and CWH, simple linear regressions were used to determine potentially relevant factors, and then multiple regression models were created. Analyses were performed with Statistica 13.5.

\section{Results}

A total of 75 patients were randomized to the study. Four patients $(5.3 \%)$ were lost to follow-up (two were lost as a result of reoperation, one was transferred to another ward, and one was excluded because of a technical problem with 
NPWT device-difficulties with maintaining airtightness), and none of those patients developed WHC or SSI within 30 days. Patient flow through the study is presented in Fig. 1 .

Table 1 shows patients' baseline characteristics before ileostomy closure. In the two study groups, 35 patients were treated with postoperative NPWT (24 males [68.6\%]; mean age $61.6 \pm 11.3$ years), and 36 patients (20 males[55.6\%]; mean age $62.4 \pm 11.3$ years) were treated with suturing of the wound and traditional dressings (control group). No significant differences between the two groups were observed in patient characteristics or preoperative treatments. Surgical outcomes are shown in Table 2, and WHC are shown in Table 3. WHC were observed in $3(8.6 \%)$ patients in the NPWT group and $11(30.6 \%)$ in the control group $(p=0.020)$. SSI was observed in two $(5.7 \%)$ patients with NPWT and in eight (22.2\%) patients in the control group $(p=0.046)$. The median LOS was $3(2-4)$ days in the NPWT group and did not significantly differ from that in the control group, 4 (2.5-5) days. The median duration of wound healing in patients with NPWT and in control groups was 7 (7-7) and $7(7-15.5)$ days, respectively $(p=0.030)$.

To identify potential risk factors for WHC, univariate logistic regression models were constructed, as presented in Table 4 . The univariate analyses revealed that only postoperative NPWT significantly decreased the odds ratio for WHC incidence. In the case of risk factors for SSI, none of the factors analyzed in the univariate regression model were statistically significant. Simple linear regression models were built for the length of hospital stay and $\mathrm{CWH}$ time. In the LOS regression model, the adjusted $R^{2}$ for multiple regression was $60.68 \%$ with a $p$ value $<0.001$. In this model, the factors identified as significantly prolonging LOS were: SSI by 4.58 days (2.62-6.54), other complications by 8.65 days $(6.20-11.10)$, and $\mathrm{BMI}<18.5 \mathrm{~kg} / \mathrm{m}^{2}$ by 7.36 days (1.22-13.50). Of all the factors analyzed in the simple regression models, only postoperative use of NPWT was significant. NPWT use was associated with shortening CWH by $3.00 \pm 1.24$ days in simple regression model with an adjusted $R^{2}$ of $6.42 \%$ ( $p$ value $<0.001$ ).

\section{Discussion}

To the best of our knowledge, this is the first study confirming the usefulness of postoperative NPWT in reducing the number of WHC associated with elective ileostomy closure in patients after surgery for colorectal cancer [14, 15]. Our study showed that the use of postoperative NPWT after ileostomy reversal procedures significantly reduces the risk of complications. This may have significant clinical implications, especially taking into considerations studies that suggest the benefits of early closure of the ileostomy, even before adjuvant chemotherapy [7]. However, concern over complications after ileostomy closure often postpones this procedure until the end of adjuvant chemotherapy. Postoperative NPWT may be an adequate solution of that clinical problem.

Although the risk of anastomotic leak after colorectal cancer surgery is reduced by diverting ileostomy, opponents argue it creates the need for another surgery with a
Fig. 1 CONSORT flow diagram. NPWT negative-pressure wound therapy

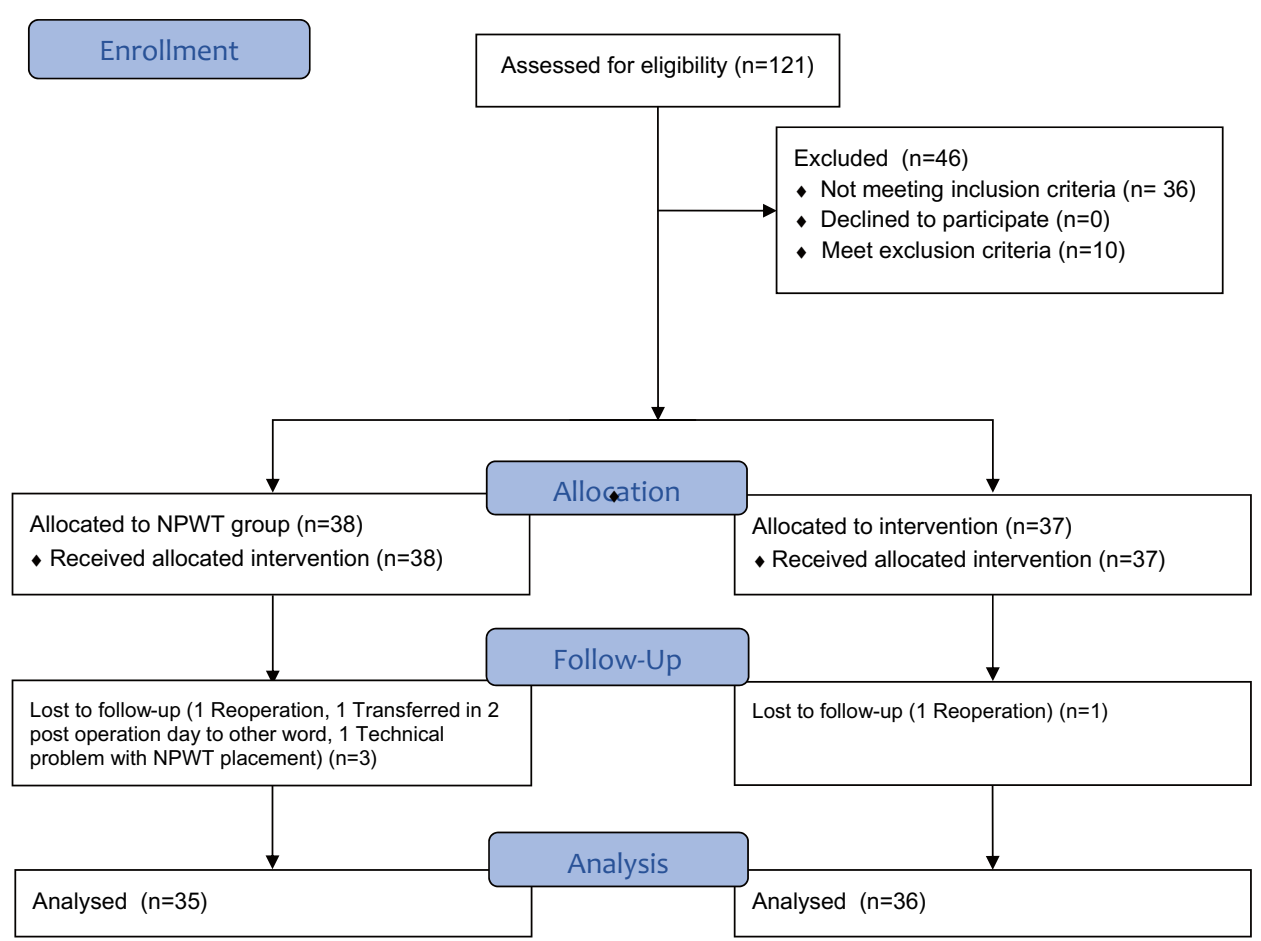


Table 1 Groups characteristics

\begin{tabular}{|c|c|c|c|}
\hline Parameter & Group 1 NPWT & Group 2 control & $p$ value \\
\hline Number of patients, $n$ & 35 & 36 & $\mathrm{n} / \mathrm{a}$ \\
\hline Females, $n(\%)$ & $11(31.4 \%)$ & $16(44.4 \%)$ & 0.259 \\
\hline Males, $n(\%)$ & $24(68.6 \%)$ & $20(55.6 \%)$ & \\
\hline Mean age, years $\pm S D$ & $61.6 \pm 11.3$ & $62.4 \pm 11.3$ & 0.974 \\
\hline Body mass index, $\mathrm{kg} / \mathrm{m}^{2} \pm \mathrm{SD}$ & $26.2 \pm 4.5$ & $26.2 \pm 4.3$ & 0.794 \\
\hline ASA $1, n(\%)$ & $1(2.9 \%)$ & $1(2.8 \%)$ & 0.943 \\
\hline ASA $2, n(\%)$ & $22(62.9 \%)$ & $24(66.7 \%)$ & \\
\hline ASA $3, n(\%)$ & $12(34.2 \%)$ & $11(30.5 \%)$ & \\
\hline Any comorbidity, $n(\%)$ & $25(71.4 \%)$ & $26(72.2 \%)$ & 0.942 \\
\hline Cardiovascular disease, $n(\%)$ & $11(31.4 \%)$ & $7(19.4 \%)$ & 0.252 \\
\hline Hypertension, $n(\%)$ & $18(51.4 \%)$ & $13(36.1 \%)$ & 0.199 \\
\hline Diabetes, $n(\%)$ & $5(14.3 \%)$ & $5(13.9 \%)$ & 0.962 \\
\hline Pulmonary disease, $n(\%)$ & $3(8.6 \%)$ & $4(11.1 \%)$ & 0.724 \\
\hline Renal disease, $n(\%)$ & $2(5.7 \%)$ & $2(5.6 \%)$ & 0.977 \\
\hline Other comorbidity, $n(\%)$ & $15(42.8 \%)$ & $14(38.9 \%)$ & 0.738 \\
\hline Smoking, $n(\%)$ & $5(14.3 \%)$ & $6(16.7 \%)$ & 0.785 \\
\hline Immunosuppressive treatment, $n(\%)$ & $3(8.6 \%)$ & $1(2.8 \%)$ & 0.297 \\
\hline Radiotherapy $n(\%)$ & $27(77.1 \%)$ & $29(80.6 \%)$ & 0.729 \\
\hline Chemotherapy (pre- or postoperative), $n(\%)$ & $27(77.1 \%)$ & $28(77.8 \%)$ & 0.950 \\
\hline AJCC Stage $0, n(\%)$ & $7(20 \%)$ & $11(30.6 \%)$ & 0.319 \\
\hline AJCC Stage I, $n(\%)$ & $7(20.0 \%)$ & $9(25.0 \%)$ & \\
\hline AJCC Stage II, $n(\%)$ & $7(20.0 \%)$ & $7(19.4 \%)$ & \\
\hline AJCC Stage III, $n(\%)$ & $13(37.1 \%)$ & $6(16.7 \%)$ & \\
\hline AJCC Stage IV, $n(\%)$ & $1(2.9 \%)$ & $3(8.3 \%)$ & \\
\hline Median time between operations, days (IQR) & $148(17-687)$ & $139(25-517)$ & 0.296 \\
\hline \multicolumn{4}{|l|}{ Previous operation } \\
\hline Hemicolectomy, $n(\%)$ & $4(11.4 \%)$ & $4(11.1 \%)$ & 0.829 \\
\hline Colectomy, $n(\%)$ & $2(5.7 \%)$ & $2(5.5 \%)$ & \\
\hline Anterior resection of rectum $n(\%)$ & $8(22.9 \%)$ & $13(36.1 \%)$ & \\
\hline Intersphincter resection $n(\%)$ & $3(8.6 \%)$ & $4(11.1 \%)$ & \\
\hline TaTME, $n(\%)$ & $18(51.4 \%)$ & $15(41.7 \%)$ & \\
\hline
\end{tabular}

$N P W T$ negative pressure wound therapy

$S D$ standard deviation

ASA American Society of Anaesthesiologists class

AJCC American Joint Committee on Cancer tumor stage

$I Q R$ inter-quartile range

TaTME transanal total mesorectum excision

relatively high risk of complications [21]. Although those tend to be relatively mild and local complications, they may cause a delay in adjuvant chemotherapy, which diminishes the results obtained by oncological treatment. One method to minimize the risk of complications after the ileostomy closure is protective NPWT placement. There is a very large divergence in the reported incidences of infectious complications after ileostomy reversal. Studies that deal with other issues related to surgery report a very low incidence of these complications [21]; however, research focusing on the impact of various surgical techniques on the incidence of SSI reports up to $40 \%$ risk of SSI in control groups. This relatively high incidence of SSI has led the American College of Surgeons (ACS) to recommend closing wounds after ileostomy reversal using the purse-string technique [10]. Our study was designed between 2015 and 2016, prior to the 2017 publication of the ACS recommendations to use the purse-string technique for closure of this type of wound [10]. Until then, simple suture technique was the standard, which is why in the control group simple suturing of the wound was applied. Because the proposed technique significantly extends healing time and often, especially in obese people, gives an unsatisfactory cosmetic effect $[9,11,12]$, many surgeons still close their ileostomy wounds with conventional 
Table 2 Peri- and postoperative outcomes in analysed groups
Table 3 Postoperative wound management complications (WMC)

\begin{tabular}{llll}
\hline Parameter & Group 1 NPWT & Group 2 control & $p$ value \\
\hline Number of patients, $n$ & 35 & 36 & n/a \\
Median operative time, minutes (IQR) & $57(50-73)$ & $55(52-70)$ & 0.856 \\
Mean perioperative blood loss, ml \pm SD & $16 \pm 9$ & $18 \pm 10$ & 0.654 \\
Patients without any complications, $n(\%)$ & $30(85.7 \%)$ & $23(63.9 \%)$ & $\mathbf{0 . 0 3 5}$ \\
Patients with complications, $n(\%)$ & $5(14.3 \%)$ & $13(36.1 \%)$ & \\
Clavien-Dindo grade 1, $n(\%)$ & $4(11.4 \%)$ & $6(16.7 \%)$ & n/a \\
Clavien-Dindo grade 2, $n(\%)$ & $1(2.9 \%)$ & $5(13.9 \%)$ & \\
Clavien-Dindo grade 3, $n(\%)$ & - & $2(5.6 \%)$ & \\
Clavien-Dindo grade 4, $n(\%)$ & - & - & \\
Clavien-Dindo grade 5, $n(\%)$ & - & - & \\
Median length of postoperative hospital stay, days (IQR) & $3(2-4)$ & $4(2.5-5)$ & 0.072 \\
Median duration of complete wound healing time days (IQR) & $7(7-7)$ & $7(7-15.5)$ & $\mathbf{0 . 0 3 0}$ \\
\hline
\end{tabular}

Significant $p$-values were marked with bolded font

$N P W T$ negative pressure wound therapy

$S D$ standard deviation

$I Q R$ inter-quartile range

\begin{tabular}{llll}
\hline Complication & Group 1 NPWT & Group 2 Control & $p$ value \\
\hline Number of WMC, $n(\%)$ & $3(8.57 \%)$ & $11(30.6 \%)$ & $\mathbf{0 . 0 2 0}$ \\
Surgical site infections (SSI), $n(\%)$ & $2(5.71 \%)$ & $8(22.2 \%)$ & $\mathbf{0 . 0 4 6}$ \\
Superficial SSI, $n(\%)$ & $2(5.71 \%)$ & $4(11.1 \%)$ & $\mathrm{n} / \mathrm{a}$ \\
Deep SSI, $n(\%)$ & 0 & $3(38.8 \%)$ & $\mathrm{n} / \mathrm{a}$ \\
Organ SSI $1, n(\%)$ & 0 & $1(2.8 \%)$ & $\mathrm{n} / \mathrm{a}$ \\
Hematoma, $n(\%)$ & 0 & $3(8.3 \%)$ & $\mathrm{n} / \mathrm{a}$ \\
Seroma, $n(\%)$ & $1(2.9 \%)$ & $1(2.8 \%)$ & 0.984 \\
\hline
\end{tabular}

Significant $p$-values were marked with bolded font primary closure [21]. The use of postoperative NPWT provides the opportunity to combine the benefits of both techniques. We suppose, based on our experience, that the healing time and final cosmetic effect do not differ from those of the primary closure technique. Additionally, NPWT offers a significant reduction in the occurrence of WMC and SSI compared with the effects of using the purse-string technique [11-13].

Several clinical trials have investigated the usefulness of postoperative NPWT in reducing postoperative infections, but the majority of them have been conducted in fields other than gastrointestinal surgery [15]. Only a few studies have been conducted in the field of general, oncological, or digestive surgery [14], and only one article has described the use of NPWT as a postoperative dressing in patients after diverting ileostomy closure [22]. The results of this study differ from our observations. In the cited study, the wound after ileostomy was closed using the purse-string suture technique; for this reason, even in the group without complications, the wound healing time was longer than 30 days [22].
In our study, even in the group of patients with an infectious complication, $\mathrm{CWH}$ was shorter than 30 days. In our research, the NPWT application was limited to $72 \mathrm{~h}$ after the surgery and was used only to evacuate the exudate or hematoma from the wound. The dressing was placed in sterile conditions of the operating room over the cleaned wound while the antibiotic prophylaxis used for the operation was still in effect. In the cited study, a NPWT was installed $24 \mathrm{~h}$ after surgery and maintained for more than 2 weeks, which in our opinion may increase the potential for colonization of the wound and possible development of infectious complications. In the previously cited meta-analysis of all other clinical trials of NPWT in the postoperative period, its use was shorter, ranging from 3 to 7 days, and the dressing was placed over the wound immediately after the surgery [15]. Another difference between our study that of Uchino et al. concerns the intervention population. Our research included patients who underwent surgery for colorectal cancer, mainly rectal cancer, which particularly exposes them to the risk of infectious complications. The majority of them 
Table 4 Univariate logistic regression analyses of WMC incidence

\begin{tabular}{lll}
\hline Parameter & OR $(95 \%$ CI $)$ & $p$ value \\
\hline Postoperative NPWT (yes vs. no) & $0.19(0.04-0.97)$ & $\mathbf{0 . 0 4 1}$ \\
Sex (female vs. male) & $0.74(0.15-3.72)$ & 0.704 \\
Age $(\geq 75$ vs. $<75$ years) & $1.72(0.16-18.91)$ & 0.650 \\
BMI ( $\geq 30$ vs. $\left.<30 \mathrm{~kg} / \mathrm{m}^{2}\right)$ & $2.07(0.33-13.18)$ & 0.429 \\
Smoking (yes vs. no) & $0.34(0.03-4.68)$ & 0.719 \\
Radiotherapy (yes vs. no) & $1.02(0.04-27.13)$ & 0.992 \\
Chemotherapy (yes vs. no) & $0.92(0.04-23.07)$ & 0.926 \\
Paraostomal hernioplasty (yes vs. no) & $0.44(0.03-5.98)$ & 0.532 \\
Time between previous operation $(\geq 365$ vs $<365$ days) & $3.08(0.23-40.65)$ & 0.381 \\
Cardiovascular disease (yes vs. no) & $0.67(0.10-4.69)$ & 0.685 \\
Diabetes (yes vs. no) & $1.44(0.19-11.13)$ & 0.719 \\
Pulmonary disease (yes vs. no) & $0.79(0.06-10.61)$ & 0.858 \\
Renal disease (yes vs. no) & $3.39(0.13-90.93)$ & 0.457 \\
TaTME (yes vs. no) & $1.25(0.25-6.34)$ & 0.780 \\
AJCC IV (yes vs. no) & $2.19(0.09-51.96)$ & 0.619 \\
Complications not related to wound management & $0.83(0.25-2.77)$ & 0.762 \\
\hline
\end{tabular}

Significant $p$-value was marked with bolded font

$W M C$ wound management complication

$N P W T$ negative pressure wound therapy

$B M I$ body mass index

TaTME transanal total mesorectum excision

AJCC American Joint Committee on Cancer tumor stage were elderly with numerous comorbidities and higher BMI. Additionally, most of them underwent radio- and chemotherapy shortly before the surgery. In the Uchino study, the population consisted of patients who had surgery as a result of ulcerative colitis, resulting in a significantly lower number of infectious complications in the control group than in our study. Given these differences, we believe that these two studies are not directly comparable, as they concern different issues. To increase the chance of achieving statistical significance while limiting the necessary study population, the cumulative index of WHC, not the occurrence of SSI, was established as the primary outcome. With the incidence of SSI in our group of patients and expected reduction of SSI cases by approximately $50 \%$, the minimal sample size would have required recruitment of 80 patients in each arm. However, the observed effect of intervention exceeded our expectations. A statistically significant effect of the use of NPWT on reducing the incidence of SSI was confirmed.

Our study showed that the use of postoperative NPWT dressing is safe. In the NPWT group, there was no increase in the percentage of postoperative complications, as well as no case of a complication that could be linked directly to NPWT use (postoperative bleeding or entero-cutaneous fistula formation). In the group of patients with NPWT, both the time of hospitalization and the time of healing of the surgical wound were shortened.

\section{Limitations}

Because of the actual incidence of WHC in this study differing from the values assumed during the sample size calculation, the post hoc analysis revealed that the study of the primary outcome, despite obtaining statistically significant differences, achieved $65 \%$ power and not the assumed $80 \%$. The obtained results are therefore underpowered.

Also, our study used only one type of NPWT device. Comparison of the effectiveness and safety of other types of NPWT equipment in this application will require further research.

We did not specifically analyze the time period between chemotherapy and operation, but we observed no difference in the incidence of postoperative chemotherapy between groups, and the time from colorectal resection and ileostomy closure until chemotherapy did not differ between groups.

We did not perform an analysis of the cosmetic effect of our treatment. Further research in this area is required.

Lastly, this study was a single-center study, which, on one hand, is a strength in the consistent treatment of all patients, but on the other hand, it will require confirmation in multicenter studies on larger groups of patients. 


\section{Conclusions}

Prophylactic postoperative NPWT after diverting ileostomy closure in colorectal cancer patients reduces the incidence of WHC, SSI, and complete wound healing time.

Acknowledgement We thank Edanz Group (https://en-author-services. edanzgroup.com/ac) for editing a draft of this manuscript.

Funding This research was financed only by the Jagiellonian University Medical College own funds, without any financial or material support from the NPWT equipment producers.

\section{Compliance with ethical standards}

Conflict of interest Authors declare no disclaimers and no conflict of interest.

Ethical approval Bioethics Committee approval No. 1072.6219.263.2019.

Informed consent Informed consent was obtained from all study participants.

Open Access This article is licensed under a Creative Commons Attribution 4.0 International License, which permits use, sharing, adaptation, distribution and reproduction in any medium or format, as long as you give appropriate credit to the original author(s) and the source, provide a link to the Creative Commons licence, and indicate if changes were made. The images or other third party material in this article are included in the article's Creative Commons licence, unless indicated otherwise in a credit line to the material. If material is not included in the article's Creative Commons licence and your intended use is not permitted by statutory regulation or exceeds the permitted use, you will need to obtain permission directly from the copyright holder. To view a copy of this licence, visit http://creativecommons.org/licenses/by/4.0/.

\section{References}

1. Qiu Y, Liu Q, Chen G et al (2016) Outcome of rectal cancer surgery in obese and nonobese patients: a meta-analysis. World J Surg Oncol. https://doi.org/10.1186/s12957-016-0775-y

2. Martinez-Perez A, Carra MC, Brunetti F, de'Angelis N (2017) Short-term clinical outcomes of laparoscopic vs open rectal excision for rectal cancer: a systematic review and meta-analysis. World J Gastroenterol 23:7906-7916. https://doi.org/10.3748/ wjg.v23.i44.7906

3. Edwards JR, Peterson KD, Mu Y et al (2009) National Healthcare Safety Network (NHSN) report: data summary for 2006 through 2008, issued December 2009. Am J Infect Control 37:783-805. https://doi.org/10.1016/j.ajic.2009.10.001

4. Detering R, Roodbeen SX, van Oostendorp SE et al (2019) Three-year nationwide experience with transanal total mesorectal excision for rectal cancer in the netherlands: a propensity score-matched comparison with conventional laparoscopic total mesorectal excision. J Am Coll Surg 228:235-244.e1. https://doi. org/10.1016/j.jamcollsurg.2018.12.016

5. Borstlap WAA, Westerduin E, Aukema TS et al (2017) Anastomotic leakage and chronic presacral sinus formation after low anterior resection: results from a large cross-sectional study. Ann
Surg 266:870-877. https://doi.org/10.1097/SLA.0000000000 002429

6. Phan K, Oh L, Ctercteko G et al (2019) Does a stoma reduce the risk of anastomotic leak and need for re-operation following low anterior resection for rectal cancer: systematic review and metaanalysis of randomized controlled trials. J Gastrointest Oncol 10:179-187. https://doi.org/10.21037/jgo.2018.11.07

7. Kłęk S, Pisarska M, Milian-Ciesielska K et al (2018) Early closure of the protective ileostomy after rectal resection should become part of the Enhanced Recovery After Surgery (ERAS) protocol: a randomized, prospective, two-center clinical trial. Wideochirurgia $\mathrm{i}$ inne Tech maloinwazyjne $=$ Videosurgery other miniinvasive Tech 13:435-441. https://doi.org/10.5114/wiitm.2018.79574

8. Perez Dominguez L, Garcia Martinez MT, Caceres Alvarado N et al (2014) Morbidity and mortality of temporary diverting ileostomies in rectal cancer surgery. Cir Esp 92:604-608. https://doi. org/10.1016/j.ciresp.2013.12.011

9. O'Leary DP, Carter M, Wijewardene D et al (2017) The effect of purse-string approximation versus linear approximation of ileostomy reversal wounds on morbidity rates and patient satisfaction: the "STOMA" trial. Tech Coloproctol 21:863-868. https://doi. org/10.1007/s10151-017-1713-x

10. Ban KA, Minei JP, Laronga $C$ et al (2017) Executive summary of the American college of surgeons/surgical infection society surgical site infection guidelines-2016 update. Surg Infect (Larchmt) 18:379-382. https://doi.org/10.1089/sur.2016.214

11. Rondelli F, Franco L, Balzarotti Canger RC et al (2018) Pursestring closure versus conventional primary closure of wound following stoma reversal: meta-analysis of randomized controlled trials. Int J Surg 52:208-213. https://doi.org/10.1016/j. ijsu.2018.02.027

12. Banerjee A (1997) Pursestring skin closure after stoma reversal. Dis Colon Rectum 40:993-994. https://doi.org/10.1007/bf020 51210

13. Reid K, Pockney P, Pollitt $\mathrm{T}$ et al (2010) Randomized clinical trial of short-term outcomes following purse-string versus conventional closure of ileostomy wounds. Br J Surg. https://doi. org/10.1002/bjs.7151

14. Webster J, Liu Z, Norman G et al (2019) Negative pressure wound therapy for surgical wounds healing by primary closure. Cochrane Database Syst Rev 3:CD009261. https://doi.org/10.1002/14651 858.CD009261.pub4

15. Strugala V, Martin R (2017) Meta-analysis of comparative trials evaluating a prophylactic single-use negative pressure wound therapy system for the prevention of surgical site complications. Surg Infect (Larchmt) 18:810-819. https://doi.org/10.1089/ sur.2017.156

16. Random number generator at https://www.naukowiec.org/kalku latory/liczby-losowe.html. Accessed 1 Jan 2016

17. O'Leary DP, Peirce C, Anglim B et al (2017) Prophylactic negative pressure dressing use in closed laparotomy wounds following abdominal operations: a randomized, controlled, open-label trial: the P.I.C.O. Trial Ann Surg 265:1082-1086. https://doi. org/10.1097/SLA.0000000000002098

18. Pedziwiatr M, Kisialeuski M, Wierdak M et al (2015) Early implementation of Enhanced Recovery After Surgery (ERAS(R)) protocol-compliance improves outcomes: a prospective cohort study. Int J Surg 21:75-81. https://doi.org/10.1016/j.ijsu.2015.06.087

19. Mangram AJ, Horan TC, Pearson ML et al (1999) Guideline for prevention of surgical site infection, 1999. centers for disease control and prevention (CDC) Hospital Infection Control Practices Advisory Committee. Am J Infect Control 27:97-132

20. Horan TC, Gaynes RP, Martone WJ et al (1992) CDC definitions of nosocomial surgical site infections, 1992: a modification of 
CDC definitions of surgical wound infections. Infect Control Hosp Epidemiol 13:606-608

21. Madani R, Day N, Kumar L et al (2019) Hand-sewn versus stapled closure of loop ileostomy: a meta-analysis. Dig Surg 36:183-194. https://doi.org/10.1159/000487310

22. Uchino M, Hirose K, Bando T et al (2016) Randomized controlled trial of prophylactic negative-pressure wound therapy at ostomy closure for the prevention of delayed wound healing and surgical site infection in patients with ulcerative colitis. Dig Surg 33:449-454. https://doi.org/10.1159/000446550

Publisher's Note Springer Nature remains neutral with regard to jurisdictional claims in published maps and institutional affiliations. 